\title{
Mortalidade por causas externas no estado de São Paulo: Uma análise epidemiológica do período de 2000 a 2018
}

\author{
Mortality from external causes in the state of São Paulo: An epidemiological analysis of the period
}

from 2000 to 2018

Mortalidad por causas externas en el estado de São Paulo: Un análisis epidemiológico del período

2000 a 2018

Recebido: 23/07/2021 | Revisado: 27/07/2021 | Aceito: 28/07/2021 | Publicado: 04/08/2021

\author{
Isabella Longhini Paiva \\ ORCID: https://orcid.org/0000-0002-3238-7348 \\ Faculdade São Leopoldo Mandic, Brasil \\ E-mail: isabella.longhini@hotmail.com \\ Márcia Regina Campos Costa da Fonseca \\ ORCID: https://orcid.org/0000-0001-5131-5561 \\ Faculdade São Leopoldo Mandic, Brasil \\ E-mail: marcia.fonseca@slmandic.edu.br
}

\begin{abstract}
Resumo
O objetivo deste estudo foi analisar a tendência e o perfil epidemiológico da mortalidade por causas externas no estado de São Paulo, no período de 2000-2018. Estudo ecológico de série temporal, sendo os dados de óbitos por causas externas (capítulo XX - CID 10) extraídos do Sistema de Informação sobre Mortalidade, sendo investigados: idade, sexo, raça, escolaridade, categoria do óbito e local de ocorrência. As variações da mortalidade por causas externas foram verificadas por meio das taxas de mortalidade padronizadas por sexo/faixa etária, estimou-se modelos de regressão linear simples para as taxas em função do ano. Para analisar se as proporções de óbitos diferiam entre estas variáveis investigadas foi utilizado o teste qui-quadrado, nível significância de 5\%. No período de estudo, ocorreram no estado 506.374 óbitos por causas externas, com diminuição significativa ao longo dos anos, taxa geral de 90,61/100.000 hab. (2000) para 50,21/100.000 hab. (2018). A queda foi observada em todos os grupos etários, em ambos os sexos, exceção aos idosos, sendo mais expressiva no sexo masculino de 15 a 29 anos $(258,82 / 100.000$ hab. 2000 para 83,65/100.000 hab. 2018). Brancos concentraram a maioria dos óbitos (81,1\%-total), notou-se aumento na proporção de óbitos em pessoas com 8 anos ou mais de escolaridade e em viúvos/separados. Quedas, acidentes de transporte terrestre, riscos acidentais à respiração e suicídio aumentaram proporcionalmente e homicídios decaíram ao longo dos anos. A maioria dos óbitos ocorreu em hospitais. Faz-se necessário conhecer a tendência e distribuição da mortalidade por causas externas para melhor direcionamento das Políticas Públicas.
\end{abstract}

Palavras-chave: Mortalidade; Causas externas; Estudos de séries temporais; Epidemiologia.

\begin{abstract}
The aim of this study was to analyze the trend and epidemiological profile of mortality from external causes in the state of São Paulo, from 2000 to 2018. Ecological time series study, with data on deaths from external causes (chapter XX ICD 10) extracted from the Mortality Information System. Age, sex, race, education, type of death and place of occurrence were investigated. Variations in mortality from external causes were verified using standardized mortality rates by sex/age group, and simple linear regression models were estimated for rates per year. To analyze whether the rate of deaths differed between the investigated variables, the chi-square test was used, significance level of 5\%. During the study period, there were 506.374 deaths from external causes in the state, with a significant decrease over the years, an overall rate of 90.61/100.000 inhabitants (2000) to 50.21/100.000 inhabitants (2018). The decrease was observed in all age groups, in both sexes, except for the elderly, being more expressive in males, 15 to 29 years old (258.82/100.000 inhabitants 2000 to 83.65/100.000 inhabitants 2018). White population concentrated the majority of deaths $(81.1 \%$ total), there was an increase in the rate of deaths for people with 8 years of schooling or more and for widowed/divorced people. Falls, land transport accidents, accidental risks to breathing and suicide have increased proportionately, and homicides have declined over the years. Most deaths occurred in hospitals. It is necessary to know the trend and distribution of mortality from external causes in order to better guide Public Policies.
\end{abstract}

Keywords: Mortality; External causes; Time series studies; Epidemiology. 


\begin{abstract}
Resumen
El objetivo de este estudio fue analizar la tendencia y el perfil epidemiológico de la mortalidad por causas externas en el estado de São Paulo, en el periodo de 2000-2018. Estudio ecológico temporal, con datos de óbitos (capítulo XX CID 10) extraídos del Sistema de Información sobre Mortalidad, investigando: edad, sexo, raza, escolaridad, categoría del óbito y lugar de ocurrencia. Las variaciones de mortalidad fueron verificadas por medio de las tasas estandarizadas por sexo/franja etaria. Se estimó modelos de regresión linear simple para las tasas en función del año. Para analizar si las proporciones de óbitos diferían entre estas variables investigadas, se utilizó el test chi-cuadrado, nivel significancia de 5\%. Durante el estudio, ocurrieron 506.374 óbitos, disminuyendo cada año, tasa general de 90,61/100.000 habs. (2000) para 50,21/100.000 habs. (2018). Se observó la baja en todas las edades, en ambos sexos, excepto a los ancianos, siendo más expresiva en el sexo masculino de 15 a 29 años $(258,82 / 100.000$ habs. en 2000 para $83,65 / 100.000$ habs.). Blancos concentran la mayoría de los óbitos (81,1\%-total), se notó aumento en personas con 8 años o más de escolaridad y en viudos/separados. Caídas, accidentes de transporte terrestre, riesgos accidentales a la respiración y suicidio aumentaron proporcionalmente y homicidios decayeron esos años. La mayoría de los óbitos ocurrió en hospitales. Es necesario conocer la tendencia y distribución de la mortalidad para mejor direccionamiento de las Políticas Públicas.
\end{abstract}

Palabras clave: Mortalidad; Causas externas; Estudios de series temporales; Epidemiología.

\title{
1. Introdução
}

A mortalidade por causas externas é um dos maiores problemas de saúde pública em todo o mundo. Esta compreende diversos tipos de ocorrências, podendo ser traumatismos, lesões ou quaisquer outros agravos à saúde intencionais ou não de início súbito e que têm como consequência imediata violência ou outra causa exógena. Nesse grupo, incluem-se as lesões provocadas no transporte, homicídios, agressões, quedas, afogamentos, envenenamentos, suicídios, queimaduras, lesões por deslizamento ou enchente e outras ocorrências provocadas por circunstâncias ambientais (Organização Mundial da Saúde, 2000).

A abordagem desse evento é complexa, uma vez que vários fatores podem estar envolvidos, tais como, socioculturais, socioeconômicos, físicos, psicológicos, de acesso a bens e serviços de saúde e segurança pública, entre outros (Messias, Bandeira, Lopes, Silva, Curado, 2018; Cardoso, Gaertner, Haritsch, Henning, Kropiwiec, Franco, 2020).

Segundo dados da Organização Mundial de Saúde, quase um terço das 5,8 milhões de mortes por lesões são resultado de violência (suicídio, homicídio e guerra) e quase um quarto é resultado de acidentes de trânsito. Outras principais causas de morte por lesões são quedas, afogamentos, queimaduras e envenenamento. Acidentes de trânsito, homicídio e suicidio, se configurarão entre as 20 causas de morte no mundo até 2030 (World Health Organization, 2014).

As causas externas desempenham papel importante na mortalidade adulta em países da América Latina, tornando-se a causa que mais mata homens adultos na região (Calazans \& Queiroz, 2020). No Chile de 1997 a 2014 ocorreram 462.442 mortes de pessoas entre 15 a 64 anos, destas 22,1\% foram por causas externas, sendo maior em homens em todos os grupos etários (85,3\% do total de mortes por causas externas) (San Martín Campos, Ponce Castro, Proboste Naranjo, YohannessenVásquez, 2019). Na Colômbia, em estudo realizado de 1998 a 2015, nos dezoito anos do estudo, registrou-se uma perda de 31.408.258 anos potenciais de vida (APPS), devido a causas externas que poderiam ser evitadas com medidas de saúde pública (média anual de 1.744.903 anos). Destes, 60,7\% (19.075.749 APPs) foram por homicídio, 17,8\% (5.579.439 APVP) para acidentes de trânsito, 15,2\% (4.781.440 APVP) de outras causas externas e 6,3\% (1.971.630 APVP) de suicídios (SeguraCardona \& Cardona-Arango, 2018).

No Brasil os indicadores não são diferentes, em 2019, dos 1.349.801 óbitos registrados no país, 142.800 (10,6\%) foram por causas externas ( $4^{\mathrm{a}}$ causa no total de óbitos), destes, 30,8\% foram por agressões e 23,0\% por acidentes de transporte (Ministério da Saúde, 2019). Em estudo realizado de 2004 a 2013, 12,58\% dos óbitos do país foram relacionados à causas externas, porém, ao considerar somente a faixa etária de 1 a 49 anos, estas apareceram como primeiro lugar nas causas de óbitos (41,12\% de todos os óbitos ocorridos nessa faixa etária) (Marques, de Souza, Vaz, Pelegrini, Linch, 2017). As causas 
externas impactam nas mortes prematuras e incapacidades, principalmente entre jovens e homens, constituindo um problema prioritário no país (Malta, Minayo, Soares Filho, da Silva, Montenegro, Ladeira, Naghavi, 2017).

De forma geral, as causas básicas da morbimortalidade por acidentes e violência em nosso país, encontram-se ligadas aos alarmantes níveis de desigualdade, pobreza, impunidade e descontrole do tráfico de armas e drogas, assim como à aparente fragilização dos valores sociais de justiça, solidariedade, igualdade de oportunidades e da esperança (Ministério da Saúde, 2003).

Algumas iniciativas governamentais têm sido realizadas para prevenção e controle desta problemática, nos últimos vinte anos destaca-se: a Política Nacional de Redução da Morbimortalidade por Acidentes e Violência (2001), tendo como propósito fundamental a redução da morbimortalidade por acidentes e violência no país, mediante o desenvolvimento de um conjunto de ações articuladas e sistematizadas, de modo a contribuir para a qualidade de vida da população; na área da mulher, a Política Nacional de Atenção Integral a Saúde da Mulher (2004), a Lei 11.340 de 2006 (Maria da Penha); na área da criança e adolescente, marcos legais de proteção, Leis 12.015/2009 e 13.010/2014, a instituição da "Linha de Cuidado para a Atenção Integral à Saúde de Crianças, Adolescentes e suas Famílias em Situação de Violências "; na área do idoso: a atualização da Política Nacional de Saúde da Pessoa Idosa (Normativa 2.528 de 2006) (Minayo, de Souza, da Silva, de Assis, 2018); além de outras iniciativas para reduzir homicídios, como, o Programa Primeiro Emprego e o Programa Bolsa Família, que objetivam manter as crianças e os jovens na escola; a criação da Força Nacional de Segurança Pública (2004), para tratar da violência urbana e reforçar a presença do Estado nas regiões com altas taxas de criminalidade; o Programa Nacional de Segurança Pública com Cidadania, para ligar as estritas políticas de segurança às medidas sociais preventivas em projetos para mulheres em risco e jovens em conflito com a lei (2008); em relação aos óbitos e lesões relacionadas ao trânsito, várias atualizações no Código Nacional de Trânsito e em 2008 a Lei 11.705 (Lei Seca), que alterou o teor legalmente permitido de álcool no sangue para zero (Reichenheim, de Souza, Moraes, de Mello Jorge, da Silva, Minayo, 2011).

Para prevenção e controle da morbimortalidade por causas externas se faz necessário investimentos de diversas áreas, não só do setor saúde, importante também uma adequada gestão da informação em saúde, bem como, qualificação das fontes de dados (declaração de óbito, autorização de internação hospitalar, notificação de violência interpessoal e autoprovocada etc.) para produção de indicadores de saúde que possam cumprir seus objetivos de basearem a tomada de decisões de gestores e profissionais de saúde (Ribeiro \& Njaine, 2020).

A realização de diagnósticos situacionais sobre a problemática, o conhecimento do perfil epidemiológico (população de maior vulnerabilidade, local de maior ocorrência, tendência ao longo do tempo), é fundamental para subsidiar o planejamento de ações de prevenção e controle da mortalidade por causas externas.

Diante do exposto este estudo teve como objetivo analisar a tendência e o perfil epidemiológico da mortalidade por causas externas no Estado de São Paulo, no período de 2000 a 2018.

\section{Metodologia}

Trata-se de um estudo ecológico de série temporal, de abordagem quantitativa, que analisou a mortalidade por causas externas no estado de São Paulo, nos anos de 2000 a 2018. Nos estudos ecológicos, compara-se a ocorrência da doença ou condição relacionada à saúde e a exposição de interesse entre agregados de indivíduos (populações de países, regiões ou municípios etc.) para verificar a possível existência de associação entre elas (Lima-Costa \& Barreto, 2003). Os dados foram extraídos do Sistema de Informação sobre Mortalidade (SIM) disponibilizado pelo tabulador de dados (TABNET) da Secretaria de Saúde do Estado de São Paulo, onde foram selecionados os óbitos cuja causa básica tenha sido causas externas, capítulo XX, da Classificação Estatística Internacional de Doenças e Problemas Relacionados à Saúde - 10ª Revisão - CID 10 
(Organização Mundial da Saúde, 2000). As variáveis investigadas foram: faixa etária, sexo, raça/cor, escolaridade, estado civil, local de ocorrência e tipo de causas. Os tipos de causas foram as disponibilizadas no sistema, a saber: atropelamento de pedestre, ciclista traumatizado em um acidente de transporte, motociclista traumatizado em um acidente de transporte, demais acidentes de trânsito e de transporte - todos estes englobados em acidentes de transportes terrestres (V01-V89), queda acidental (W00-W19), afogamento acidental (W65-W67), outros riscos acidentais relacionados à respiração (W75-W84), choque elétrico acidental (W87), exposição a fogo acidental (X00-X09), lesões autoprovocadas intencionalmente/suicídio (X60-X84), agressões/homicídio (X85-Y09), acidentes não especificados/lesões de intenção indeterminada (Y10-Y34) e demais causas externas.

Para verificar as variações da mortalidade por causas externas nos períodos de estudo (2000 a 2018) inicialmente analisou-se as taxas brutas de mortalidade por 100.000 hab. por ano, estas calculadas levando-se em consideração o número de óbitos por causas externas da população residente no estado dividido pela população residente do estado (Rede Interagencial de Informações para a Saúde-RIPSA, 2008). A quantificação da população residente (denominador das taxas) se deu por meio dos dados extraídos do TABNET da Secretaria de Saúde do Estado de São Paulo - item "Demografia", sendo estas estimativas censitárias fornecidas pela Fundação Sistema Estadual de Análises de Dados (SEADE). O sistema fornece dados da população por sexo e faixa etária.

A seguir, padronizou-se as taxas de óbitos por sexo, faixa etária, sexo/faixa etária, pelo método direto ou de base fixa, considerando como referência, a população brasileira obtida no censo de 2010. Este ajuste é necessário para minimizar o efeito de diferenças etárias e de sexo na população em períodos distintos, a fim de que diferenças temporais não possam ser atribuídas a estas características.

Posteriormente realizou-se análises de regressão linear simples para as taxas brutas e padronizadas, em função do ano. Estimou-se modelos de regressão $\mathrm{Y}=\alpha+\beta \mathrm{X}$, onde $\mathrm{Y}$ é a variável de resposta (óbitos), $\mathrm{X}$ é o ano, $\alpha$ é o coeficiente linear, $\mathrm{o}$ intercepto, ou seja, o ponto onde a reta corta o eixo das ordenadas (eixo vertical) e $\beta$ é a inclinação da reta. Testou-se as hipóteses nulas $\beta=0$, sabendo-se que um $\beta$ estatisticamente significativo e positivo indica tendência de crescimento do óbito no tempo e um $\beta$ estatisticamente significativo e negativo indica tendência de decréscimo do óbito no tempo. Calculou-se também os coeficientes de determinação dos modelos, ou seja, o quanto da variação na quantidade de óbitos é explicada pelo modelo de regressão estimado. Esta medida pode variar de zero a um e é utilizada como uma indicação da adequação do modelo de regressão.

Também foram apresentadas as distribuições de frequência (absoluta e percentual) da mortalidade por causas externas segundo as variáveis: faixa etária, sexo, raça/cor, escolaridade, estado civil, local de ocorrência e tipo de causas. Para analisar se as proporções de óbitos por causas externas diferiam entre estas variáveis foi utilizado o teste qui-quadrado para aderência. As hipóteses nulas testadas foram $\mathrm{H} 0: \mathrm{p} 1=\mathrm{p} 2, \ldots ., \mathrm{pk}$, sendo $\mathrm{p}$ a probabilidade de cada categoria $\mathrm{i}, \mathrm{i}=1, \ldots, \mathrm{k}$. As análises foram realizadas no programa $\mathrm{R}$ (Core Team, 2018), com nível de significância de 5\%.

Os dados do SIM encontram-se divulgados e disponibilizados no TABNET, portanto, são de domínio público e não permitem o conhecimento da identidade das vítimas cujos registros constam dos bancos analisados, portanto, este projeto obteve dispensa do Comitê de Ética em Pesquisa da Faculdade São Leopoldo Mandic (№ 2019/0198).

\section{Resultados}

Durante os anos de 2000 a 2018 foram registrados no estado de São Paulo 506.374 óbitos por causas externas. As taxas de óbitos vêm apresentando tendência de queda (Tabela 1). As taxas não padronizadas passaram de 90,61 para 50,21 em 100.000 habitantes, a padronizada por sexo de 90,58 para 50,35 em 100.000 habitantes, a padronizada por faixa etária de 89,58 
para 45,81 em 100.000 habitantes e a padronizada por sexo e idade de 89,93 óbitos em 2000 para 45,77 óbitos em 2018 por 100.000 habitantes.

Tabela 1. Taxas de óbitos (óbitos por 100.000 habitantes) por causas externas no estado de São Paulo de 2000 a 2018.

\begin{tabular}{|c|c|c|c|c|}
\hline \multirow[b]{2}{*}{ Ano } & \multicolumn{4}{|c|}{ Taxas (óbitos por 100.000 habitantes) } \\
\hline & Padronizada para sexo & $\begin{array}{c}\text { Padronizada para faixa } \\
\text { etária }\end{array}$ & $\begin{array}{c}\text { Padronizada para sexo e } \\
\text { faixa etária }\end{array}$ & Não padronizada \\
\hline 2000 & 90,58 & 89,58 & 89,93 & 90,61 \\
\hline 2001 & 89,57 & 88,85 & 89,02 & 89,60 \\
\hline 2002 & 85,31 & 84,56 & 84,52 & 85,28 \\
\hline 2003 & 80,75 & 80,27 & 79,93 & 80,69 \\
\hline 2004 & 74,89 & 74,88 & 74,05 & 74,82 \\
\hline 2005 & 69,01 & 68,19 & 68,03 & 68,92 \\
\hline 2006 & 66,92 & 66,36 & 65,75 & 66,82 \\
\hline 2007 & 64,60 & 61,14 & 63,13 & 64,48 \\
\hline 2008 & 63,06 & 60,94 & 61,28 & 62,89 \\
\hline 2009 & 63,01 & 60,50 & 60,73 & 62,85 \\
\hline 2010 & 61,06 & 58,05 & 58,24 & 60,86 \\
\hline 2011 & 58,39 & 55,41 & 55,30 & 58,22 \\
\hline 2012 & 60,62 & 57,52 & 57,09 & 60,43 \\
\hline 2013 & 57,44 & 53,80 & 53,85 & 57,28 \\
\hline 2014 & 59,52 & 55,72 & 55,74 & 59,36 \\
\hline 2015 & 55,04 & 51,10 & 51,11 & 54,88 \\
\hline 2016 & 53,91 & 49,68 & 49,67 & 53,77 \\
\hline 2017 & 53,79 & 49,38 & 49,35 & 53,64 \\
\hline 2018 & 50,35 & 45,81 & 45,77 & 50,21 \\
\hline \multicolumn{5}{|l|}{ Regressão linear } \\
\hline Coeficiente linear & $4.221,12$ & $4.726,93$ & $4.732,62$ & $4.239,97$ \\
\hline Coeficiente angular & $-2,07$ & $-2,32$ & $-2,32$ & $-2,08$ \\
\hline $\mathrm{R}^{2}$ & 0,8882 & 0,9115 & 0,9167 & 0,8876 \\
\hline p-valor & $<0,0001$ & $<0,0001$ & $<0,0001$ & $<0,0001$ \\
\hline
\end{tabular}

Fonte: Sistema de Informação sobre Mortalidade (SIM), TABNET, São Paulo.

A proporção de óbitos por causas externas foi maior no sexo masculino (81,1\% - no total de óbitos), 85,8\% em 2000 e 76,1\% em 2018, porém, observou-se aumento dos óbitos na população feminina, de 14,2\% em 2000 para 23,8\% em 2018, ressalta-se que, ao longo da série histórica a proporção de homens e mulheres no estado de São Paulo, se manteve constante (em torno de $50 \%)$.

A proporção de óbitos por faixa etária foi maior entre os indivíduos de 15 a 29 anos (32,9\% do total) e de 30 a 49 anos (31,8\% do total), porém, ao longo dos anos, observou-se diminuição nestas faixas etárias, mais expressiva nos indivíduos de 15 a 29 anos (menos de 15 anos de 5,3\% em 2000 para 2,9\% em 2018; de 15 a 29 anos de 43,6\% em 2000 para 22,5\% em 2018, de 30 a 49 anos de 32,1\% em 2000 para 29,9\% em 2018), e aumento nas faixas etárias acima dos 50 anos (de 50 a 69 anos de 10,9\% em 2000 para 21,0\% em 2018; de 70 anos ou mais de 5,8\% para 22,4\% em 2018 (p<0,0001), cabe ressaltar que a distribuição etária da população de São Paulo também variou ao longo do tempo, com um aumento de pessoas mais velhas (principalmente de 50 anos ou mais).

$\mathrm{Na}$ análise proporcional conjunta sexo/faixa etária, notou-se que o número de óbitos diminuiu ao longo do tempo, tanto para homens, quanto para mulheres, principalmente até 29 anos de idade (até 29 anos - homens 43,3\% 2000/ 21,4\% 2018 - mulheres 5,7\% 2000/4,1\% 2018), enquanto aumentou para os dois sexos com 50 anos ou mais (50 anos ou mais - homens $12,0 \% 2000 / 28,2 \% 2018$ - mulheres 4,8\% 2000/15,3\% 2018) $(\mathrm{p}<0,0001)$. 
Para as variáveis, faixa etária e sexo, pela existência de dados censitários da população, estratificada por estes parâmetros, foi possível analisar as taxas de mortalidade ao longo da série histórica (2000-2018).

Embora, em todos os anos do estudo (2000-2018) as taxas de óbitos sejam maiores entre os homens, observou-se queda significativa destas, passando de 158,79 para 78,56 óbitos a cada 100.000 habitantes ( $<<0,0001$ ), já a taxa do sexo feminino não apresentou variação significativa no tempo ( $\mathrm{p}$-valor $=0,1829$ ), passando de 25,14 para 23,29 óbitos a cada 100.000 habitantes.

Em relação as taxas de óbitos por faixas etárias houve maior tendência de queda entre as pessoas de 15 a 29 anos passando de 140,72 (2000) para 48,87 óbitos (2018) a cada 100.000 habitantes (coeficiente angular: -4,71; p<0,0001). Exceção aos iguais ou maiores de 70 anos, também se observou diminuição nas taxas, em outras faixas etárias, porém menos expressivas, menores de 15 anos de 18,34 em 2000 para 7,72 em 2018 a cada 100.000 habitantes (coeficiente angular: -0,46; p<0,0001), de 30 a 49 anos de 101,27 em 2000 para 48,03 em 2018 a cada 100.000 habitantes (coeficiente angular: $-2,76 ; \mathrm{p}<$ 0,0001), de 50 a 69 anos de 75,28 em 2000 para 52,04 em 2018 a cada 100.000 habitantes (coeficiente angular: -1,46; p< 0,0001). Os indivíduos de 70 anos ou mais apresentaram aumento nas taxas de óbitos passando de 136,63 (2000) para 185,07 (2018) a cada 100.000 habitantes (coeficiente angular: $+1,50, \mathrm{p}=0,0394$ ).

Quando analisados as taxas de óbitos por sexo/faixa etária conjuntamente, ambos os sexos apresentaram tendências de diminuição ao longo do tempo, exceto pela faixa etária de 70 anos ou mais (masculino - 179,13 em 2000 para 229,08 em 2018 a cada 100.000 habitantes, coeficiente angular $=+2,17, \mathrm{p}=0,0020$; feminino $-107,11 \mathrm{em} 2000$ para 155,46 em 2018 a cada 100.000 habitantes, coeficiente angular $=+2,30, \mathrm{p}=0,0018$ ). O sexo feminino apresentou menos da metade da magnitude do sexo masculino, em todos os casos. A maior tendência de queda foi observada no sexo masculino de 15 a 29 anos, de 258,82 em 2000 para 83,65 em 2018 a cada 100.000 habitantes (coeficiente angular: -9,36, p<0,0001) (Tabela 2). 
Research, Society and Development, v. 10, n. 9, e15101018627, 2021

(CC BY 4.0) | ISSN 2525-3409 | DOI: http://dx.doi.org/10.33448/rsd-v10i10.18627

Tabela 2. Taxas de óbitos (óbitos por 100.000 habitantes) por causas externas no estado de São Paulo em função do sexo e da faixa etária.

\begin{tabular}{|c|c|c|c|c|c|c|c|c|c|c|}
\hline \multirow[b]{2}{*}{ Ano } & \multicolumn{4}{|c|}{ Masculino } & \multirow[b]{2}{*}{$\begin{array}{c}70 \text { anos ou } \\
\text { mais }\end{array}$} & \multirow[b]{2}{*}{$\begin{array}{c}\text { Menos de } 15 \\
\text { anos }\end{array}$} & \multicolumn{4}{|c|}{ Feminino } \\
\hline & $\begin{array}{c}\text { Menos de } 15 \\
\text { anos }\end{array}$ & $\begin{array}{c}\text { De } 15 \text { a } 29 \\
\text { anos }\end{array}$ & $\begin{array}{c}\text { De } 30 \text { a } 49 \\
\text { anos }\end{array}$ & $\begin{array}{c}\text { De } 50 \text { a } 69 \\
\text { anos }\end{array}$ & & & $\begin{array}{c}\text { De } 15 \text { a } 29 \\
\text { anos }\end{array}$ & $\begin{array}{c}\text { De } 30 \text { a } 49 \\
\text { anos }\end{array}$ & $\begin{array}{c}\text { De } 50 \text { a } 69 \\
\text { anos }\end{array}$ & $\begin{array}{c}70 \text { anos ou } \\
\text { mais }\end{array}$ \\
\hline 2000 & 23,62 & 258,82 & 185,53 & 129,73 & 179,13 & 12,96 & 24,39 & 21,96 & 27,13 & 107,11 \\
\hline 2001 & 21,74 & 252,65 & 185,47 & 131,73 & 203,54 & 11,17 & 23,49 & 20,34 & 25,45 & 115,11 \\
\hline 2002 & 19,88 & 244,00 & 171,64 & 123,55 & 192,92 & 10,49 & 23,73 & 19,44 & 24,51 & 111,21 \\
\hline 2003 & 18,38 & 225,06 & 162,06 & 121,75 & 191,38 & 9,66 & 22,51 & 18,44 & 24,17 & 113,68 \\
\hline 2004 & 17,91 & 190,75 & 149,46 & 119,18 & 208,92 & 9,96 & 20,93 & 18,51 & 24,06 & 133,17 \\
\hline 2005 & 17,76 & 158,17 & 137,65 & 120,67 & 207,89 & 10,38 & 20,98 & 18,82 & 25,76 & 122,11 \\
\hline 2006 & 17,10 & 146,78 & 130,88 & 117,07 & 223,33 & 10,18 & 19,83 & 17,45 & 25,06 & 145,57 \\
\hline 2007 & 16,16 & 133,45 & 123,25 & 119,72 & 230,66 & 8,79 & 18,42 & 17,74 & 24,75 & 157,92 \\
\hline 2008 & 14,45 & 125,63 & 120,66 & 114,26 & 237,27 & 8,53 & 16,38 & 18,00 & 25,55 & 166,40 \\
\hline 2009 & 14,40 & 122,52 & 118,92 & 114,34 & 246,85 & 8,47 & 16,97 & 17,30 & 23,96 & 169,03 \\
\hline 2010 & 12,77 & 116,06 & 113,77 & 112,52 & 241,88 & 7,54 & 17,82 & 16,39 & 23,74 & 157,59 \\
\hline 2011 & 12,59 & 114,63 & 109,46 & 106,82 & 207,36 & 7,38 & 16,94 & 16,37 & 21,71 & 132,42 \\
\hline 2012 & 12,64 & 120,00 & 114,26 & 103,85 & 217,69 & 7,29 & 17,31 & 16,71 & 23,40 & 138,71 \\
\hline 2013 & 12,47 & 108,85 & 106,84 & 102,92 & 216,61 & 7,67 & 15,34 & 15,76 & 21,78 & 135,13 \\
\hline 2014 & 12,40 & 116,52 & 109,05 & 104,54 & 218,08 & 7,82 & 16,53 & 16,10 & 21,57 & 142,12 \\
\hline 2015 & 11,23 & 102,40 & 96,49 & 97,23 & 228,41 & 6,74 & 14,47 & 14,43 & 21,25 & 147,83 \\
\hline 2016 & 10,07 & 93,30 & 92,56 & 99,34 & 235,40 & 6,33 & 14,40 & 15,44 & 19,23 & 156,84 \\
\hline 2017 & 9,59 & 93,71 & 90,95 & 97,38 & 234,24 & 6,58 & 14,53 & 14,73 & 20,76 & 157,02 \\
\hline 2018 & 9,29 & 83,65 & 84,16 & 90,79 & 229,08 & 6,08 & 13,06 & 13,53 & 18,60 & 155,46 \\
\hline \multicolumn{11}{|l|}{ Regressão Linear } \\
\hline Coeficiente linear & $1.455,72$ & $18.950,78$ & $10.788,97$ & $4.176,55$ & $-4.139,60$ & 640,95 & $1.219,41$ & 735,13 & 763,42 & $-4.481,14$ \\
\hline Coeficiente angular & $-0,72$ & $-9,36$ & $-5,31$ & $-2,02$ & 2,17 & $-0,31$ & $-0,60$ & $-0,36$ & $-0,37$ & 2,30 \\
\hline $\mathrm{R}^{2}$ & 0,9533 & 0,8423 & 0,9206 & 0,9608 & 0,4387 & 0,8997 & 0,9279 & 0,9146 & 0,8050 & 0,4468 \\
\hline p-valor & $<0,0001$ & $<0,0001$ & $<0,0001$ & $<0,0001$ & 0,0020 & $<0,0001$ & $<0,0001$ & $<0,0001$ & $<0,0001$ & 0,0018 \\
\hline
\end{tabular}

Fonte: Sistema de Informação sobre Mortalidade (SIM), TABNET, São Paulo. 
Em relação a raça/cor observou-se maior percentual de óbitos por causas externas entre os indivíduos brancos em todos os anos de estudo (65,8\% para o total), seguidos de pardos (24,7\% para o total). Comparando-se os anos de 2000 e 2018 , respectivamente, a raça/cor branca variou de $63,0 \%$ para $64,0 \%$, a preta de $6,8 \%$ para $5,5 \%$, a amarela de $1,9 \%$ para $1,1 \%$, a parda de $24,8 \%$ para $28,1 \%$ e a indígena de $0,2 \%$ para $0,0 \%$ e ignorados de $3,3 \%$ para $1,4 \%$ (p<0,0001). Vale ressaltar que no estado de São Paulo existe uma concentração maior de brancos (Fundação Sistema Estadual de Análises de Dados-SEADE, 2010).

Quanto a escolaridade, excluindo-se os ignorados, o maior percentual de óbitos (25,0\% do total) se concentrou entre os indivíduos com 4 a 7 anos de escolaridade. Notou-se aumento na proporção de óbitos em pessoas com 8 anos ou mais de escolaridade de 12,3\% em 2000 para 33,1\% em 2018 (8 a 11 anos de escolaridade de 9,6\% em 2000 para 25,8\% em 2018; 12 anos ou mais de escolaridade de 2,7\% em 2000 para 7,3\% em 2018), entretanto, também diminuiu o número de óbitos de pessoas com escolaridade desconhecida (43,0\% em 2000 para 28,9\% em 2018). (Tabela 3)

Tabela 3. Número de óbitos por causas externas no estado de São Paulo em função da escolaridade de 2000 a 2018.

\begin{tabular}{|c|c|c|c|c|c|c|c|}
\hline \multirow[b]{2}{*}{ Ano } & \multirow[b]{2}{*}{ Total } & \multicolumn{6}{|c|}{ Escolaridade } \\
\hline & & Nenhuma & De 1 a 3 anos & De 4 a 7 anos & De 8 a 11 anos & $\begin{array}{c}12 \text { anos ou } \\
\text { mais }\end{array}$ & Ignorado \\
\hline 2000 & $33501(100,0 \%)$ & $1505(4,5 \%)$ & $4945(14,8 \%)$ & $8523(25,4 \%)$ & $3201(9,6 \%)$ & $917(2,7 \%)$ & $14410(43,0 \%)$ \\
\hline 2001 & $33560(100,0 \%)$ & $1318(3,9 \%)$ & $5025(15,0 \%)$ & $8273(24,7 \%)$ & $3363(10,0 \%)$ & $934(2,8 \%)$ & $14647(43,6 \%)$ \\
\hline 2002 & $32328(100,0 \%)$ & $1391(4,3 \%)$ & $4551(14,1 \%)$ & $8527(26,4 \%)$ & $3375(10,4 \%)$ & $1023(3,2 \%)$ & $13461(41,6 \%)$ \\
\hline 2003 & $30939(100,0 \%)$ & $1374(4,4 \%)$ & $3969(12,8 \%)$ & $7999(25,9 \%)$ & $3802(12,3 \%)$ & $1161(3,8 \%)$ & $12634(40,8 \%)$ \\
\hline 2004 & $29009(100,0 \%)$ & $1419(4,9 \%)$ & $3742(12,9 \%)$ & $6846(23,6 \%)$ & $3858(13,3 \%)$ & $1179(4,1 \%)$ & $11965(41,2 \%)$ \\
\hline 2005 & $27019(100,0 \%)$ & $1394(5,2 \%)$ & $3193(11,8 \%)$ & $6082(22,5 \%)$ & $4135(15,3 \%)$ & $1001(3,7 \%)$ & $11214(41,5 \%)$ \\
\hline 2006 & $26474(100,0 \%)$ & $1313(5,0 \%)$ & $2769(10,5 \%)$ & $5014(18,9 \%)$ & $4195(15,8 \%)$ & $1032(3,9 \%)$ & $12151(45,9 \%)$ \\
\hline 2007 & $25807(100,0 \%)$ & $1346(5,2 \%)$ & $2597(10,1 \%)$ & $5125(19,9 \%)$ & $4469(17,3 \%)$ & $1193(4,6 \%)$ & $11077(42,9 \%)$ \\
\hline 2008 & $25422(100,0 \%)$ & $1368(5,4 \%)$ & $2782(10,9 \%)$ & $5707(22,4 \%)$ & $4402(17,3 \%)$ & $1112(4,4 \%)$ & $10051(39,5 \%)$ \\
\hline 2009 & $25652(100,0 \%)$ & $1442(5,6 \%)$ & $2858(11,1 \%)$ & $5627(21,9 \%)$ & $4661(18,2 \%)$ & $1266(4,9 \%)$ & $9798(38,2 \%)$ \\
\hline 2010 & $25089(100,0 \%)$ & $1390(5,5 \%)$ & $2844(11,3 \%)$ & $5769(23,0 \%)$ & $5101(20,3 \%)$ & $1317(5,2 \%)$ & $8668(34,5 \%)$ \\
\hline 2011 & $24209(100,0 \%)$ & $757(3,1 \%)$ & $3187(13,2 \%)$ & $5959(24,6 \%)$ & $5108(21,1 \%)$ & $1419(5,9 \%)$ & $7779(32,1 \%)$ \\
\hline 2012 & $25346(100,0 \%)$ & $843(3,3 \%)$ & $3264(12,9 \%)$ & $6873(27,1 \%)$ & $5519(21,8 \%)$ & $1293(5,1 \%)$ & $7554(29,8 \%)$ \\
\hline 2013 & $24231(100,0 \%)$ & $813(3,4 \%)$ & $3036(12,5 \%)$ & $6741(27,8 \%)$ & $5459(22,5 \%)$ & $1387(5,7 \%)$ & $6795(28,0 \%)$ \\
\hline 2014 & $25329(100,0 \%)$ & $843(3,3 \%)$ & $3146(12,4 \%)$ & $7393(29,2 \%)$ & $5806(22,9 \%)$ & $1405(5,5 \%)$ & $6736(26,6 \%)$ \\
\hline 2015 & $23626(100,0 \%)$ & $867(3,7 \%)$ & $3208(13,6 \%)$ & $7357(31,1 \%)$ & $5369(22,7 \%)$ & $1335(5,7 \%)$ & $5490(23,2 \%)$ \\
\hline 2016 & $23314(100,0 \%)$ & $891(3,8 \%)$ & $2948(12,6 \%)$ & $7155(30,7 \%)$ & $6018(25,8 \%)$ & $1491(6,4 \%)$ & $4811(20,6 \%)$ \\
\hline 2017 & $23428(100,0 \%)$ & $871(3,7 \%)$ & $2835(12,1 \%)$ & $6370(27,2 \%)$ & $6443(27,5 \%)$ & $1555(6,6 \%)$ & $5354(22,9 \%)$ \\
\hline 2018 & $22091(100,0 \%)$ & $868(3,9 \%)$ & $2512(11,4 \%)$ & $5011(22,7 \%)$ & $5704(25,8 \%)$ & $1607(7,3 \%)$ & $6389(28,9 \%)$ \\
\hline Total & $\begin{array}{c}506374 \\
(100,0 \%)\end{array}$ & $\begin{array}{l}22013 \\
(4,3 \%)\end{array}$ & $\begin{array}{c}63411 \\
(12,5 \%)\end{array}$ & $\begin{array}{l}126351 \\
(25,0 \%)\end{array}$ & $\begin{array}{c}89988 \\
(17,8 \%)\end{array}$ & $\begin{array}{l}23627 \\
(4,7 \%)\end{array}$ & $\begin{array}{l}180984 \\
(35,7 \%)\end{array}$ \\
\hline
\end{tabular}

$\mathrm{p}<0,0001$.

Fonte: Sistema de Informação sobre Mortalidade (SIM), TABNET, São Paulo. 
Na Tabela 4 é apresentado os resultados referentes à variável estado civil, observou-se aumento da proporção de óbitos de pessoas viúvas (4,1\% em 2000 para 10,6\% em 2018) e separadas (3,2\% em 2000 para 7,1\% em 2018), por outro lado, houve diminuição de óbitos em ralação aos solteiros (59,0\% em 2000 para 43,7\% em 2018).

Tabela 4. Número de óbitos por causas externas no estado de São Paulo em função da escolaridade de 2000 a 2018.

\begin{tabular}{|c|c|c|c|c|c|c|c|}
\hline \multirow[b]{2}{*}{ Ano } & \multirow[b]{2}{*}{ Total } & \multicolumn{6}{|c|}{ Estado civil } \\
\hline & & Solteiro & Casado & Viúvo & $\begin{array}{c}\text { Separado } \\
\text { judicialmente }\end{array}$ & $\begin{array}{c}\text { União } \\
\text { consensual }\end{array}$ & Ignorado \\
\hline 2000 & $33501(100,0 \%)$ & $19767(59,0 \%)$ & $7944(23,7 \%)$ & $1372(4,1 \%)$ & $1074(3,2 \%)$ & $814(2,4 \%)$ & $2530(7,6 \%)$ \\
\hline 2001 & $33560(100,0 \%)$ & $19578(58,3 \%)$ & $7755(23,1 \%)$ & $1566(4,7 \%)$ & $1164(3,5 \%)$ & $771(2,3 \%)$ & $2726(8,1 \%)$ \\
\hline 2002 & $32328(100,0 \%)$ & $19005(58,8 \%)$ & $7118(22,0 \%)$ & $1475(4,6 \%)$ & $1283(4,0 \%)$ & $729(2,3 \%)$ & $2718(8,4 \%)$ \\
\hline 2003 & $30939(100,0 \%)$ & $17449(56,4 \%)$ & $6556(21,2 \%)$ & $1463(4,7 \%)$ & $1126(3,6 \%)$ & $506(1,6 \%)$ & $3839(12,4 \%)$ \\
\hline 2004 & $29009(100,0 \%)$ & $16820(58,0 \%)$ & $6497(22,4 \%)$ & $1699(5,9 \%)$ & $1417(4,9 \%)$ & $52(0,2 \%)$ & $2524(8,7 \%)$ \\
\hline 2005 & $27019(100,0 \%)$ & $14997(55,5 \%)$ & $6395(23,7 \%)$ & $1703(6,3 \%)$ & $1532(5,7 \%)$ & $256(0,9 \%)$ & $2136(7,9 \%)$ \\
\hline 2006 & $26474(100,0 \%)$ & $25782(97,4 \%)$ & $3(0,0 \%)$ & $1(0,0 \%)$ & $2(0,0 \%)$ & $1(0,0 \%)$ & $685(2,6 \%)$ \\
\hline 2007 & $25807(100,0 \%)$ & $13523(52,4 \%)$ & $6227(24,1 \%)$ & $2015(7,8 \%)$ & $1503(5,8 \%)$ & $14(0,1 \%)$ & $2525(9,8 \%)$ \\
\hline 2008 & $25422(100,0 \%)$ & $13363(52,6 \%)$ & $6311(24,8 \%)$ & $2254(8,9 \%)$ & $1710(6,7 \%)$ & $18(0,1 \%)$ & $1766(6,9 \%)$ \\
\hline 2009 & $25652(100,0 \%)$ & $13608(53,0 \%)$ & $6477(25,2 \%)$ & $2361(9,2 \%)$ & $1806(7,0 \%)$ & $22(0,1 \%)$ & $1378(5,4 \%)$ \\
\hline 2010 & $25089(100,0 \%)$ & $12615(50,3 \%)$ & $8007(31,9 \%)$ & $2110(8,4 \%)$ & $1635(6,5 \%)$ & $67(0,3 \%)$ & $655(2,6 \%)$ \\
\hline 2011 & $24209(100,0 \%)$ & $11720(48,4 \%)$ & $5866(24,2 \%)$ & $1953(8,1 \%)$ & $1583(6,5 \%)$ & $344(1,4 \%)$ & $2743(11,3 \%)$ \\
\hline 2012 & $25346(100,0 \%)$ & $12229(48,2 \%)$ & $6108(24,1 \%)$ & $2030(8,0 \%)$ & $1569(6,2 \%)$ & $652(2,6 \%)$ & $2758(10,9 \%)$ \\
\hline 2013 & $24231(100,0 \%)$ & $11516(47,5 \%)$ & $6036(24,9 \%)$ & $2005(8,3 \%)$ & $1548(6,4 \%)$ & $681(2,8 \%)$ & $2445(10,1 \%)$ \\
\hline 2014 & $25329(100,0 \%)$ & $12279(48,5 \%)$ & $5920(23,4 \%)$ & $2060(8,1 \%)$ & $1614(6,4 \%)$ & $680(2,7 \%)$ & $2776(11,0 \%)$ \\
\hline 2015 & $23626(100,0 \%)$ & $11203(47,4 \%)$ & $5609(23,7 \%)$ & $2158(9,1 \%)$ & $1508(6,4 \%)$ & $737(3,1 \%)$ & $2411(10,2 \%)$ \\
\hline 2016 & $23314(100,0 \%)$ & $10669(45,8 \%)$ & $5792(24,8 \%)$ & $2308(9,9 \%)$ & $1617(6,9 \%)$ & $765(3,3 \%)$ & $2163(9,3 \%)$ \\
\hline 2017 & $23428(100,0 \%)$ & $10621(45,3 \%)$ & $5767(24,6 \%)$ & $2390(10,2 \%)$ & $1758(7,5 \%)$ & $723(3,1 \%)$ & $2169(9,3 \%)$ \\
\hline 2018 & $22091(100,0 \%)$ & $9643(43,7 \%)$ & $5368(24,3 \%)$ & $2351(10,6 \%)$ & $1563(7,1 \%)$ & $762(3,4 \%)$ & $2404(10,9 \%)$ \\
\hline Total & $\begin{array}{c}506374 \\
(100,0 \%)\end{array}$ & $\begin{array}{l}276387 \\
(54,6 \%)\end{array}$ & $\begin{array}{l}115756 \\
(22,9 \%)\end{array}$ & $\begin{array}{l}35274 \\
(7,0 \%)\end{array}$ & $\begin{array}{l}27012 \\
(5,3 \%)\end{array}$ & $\begin{array}{c}8594 \\
(1,7 \%)\end{array}$ & $\begin{array}{l}43351 \\
(8,6 \%)\end{array}$ \\
\hline
\end{tabular}

$\mathrm{p}<0,0001$.

Fonte: Sistema de Informação sobre Mortalidade (SIM), TABNET, São Paulo.

Em relação às causas (CID 10) dos óbitos notou-se que as mortes por queda acidental (W00-W19) aumentaram proporcionalmente no período em relação aos outros tipos, indo de 1,8\% do total de mortes por causas externas no estado em 2000 para 14,0\% em 2018. As mortes por acidentes de transporte terrestre, outros riscos acidentais à respiração e suicídio também aumentaram proporcionalmente de 21,8\%, 1,0\% e 4,0\% do total de mortes no estado em 2000 para 28,4\%, 3,7\% e $9,2 \%$, respectivamente. As mortes por lesões de intenção indeterminada diminuíram até 2013, voltando a subir nos anos posteriores, principalmente em 2018, passando a 18,1\% do total. Por outro lado, as mortes por acidente não especificado e homicídio diminuíram de 9,3\% e 43,8\% do total de mortes no estado em 2000 para 3,1\% e 15,0\%, respectivamente (Tabela 5). 
Research, Society and Development, v. 10, n. 9, e15101018627, 2021

(CC BY 4.0) | ISSN 2525-3409 | DOI: http://dx.doi.org/10.33448/rsd-v10i10.18627

Tabela 5. Número de óbitos por causas externas no estado de São Paulo em função da causa do óbito de 2000 a 2018.

\begin{tabular}{|c|c|c|c|c|c|c|c|c|c|c|c|c|}
\hline \multirow[b]{2}{*}{ Ano } & \multirow[b]{2}{*}{ Total } & \multicolumn{11}{|c|}{ Causa do óbito* } \\
\hline & & V01-V89 & W00-W19 & W65-W67 & W75-W84 & W87 & X00-X09 & $\begin{array}{c}\text { Y 10-Y34 } \\
\text { (acidentes) }\end{array}$ & X60-X84 & X85-Y09 & $\begin{array}{l}\text { Y10-Y34 } \\
\text { (lesões) }\end{array}$ & Outras \\
\hline 2000 & $35501(100,0 \%)$ & $7749(21,8 \%)$ & $644(1,8 \%)$ & $1334(3,8 \%)$ & $343(1,0 \%)$ & $178(0,5 \%)$ & $177(0,5 \%)$ & $3288(9,3 \%)$ & $1408(4,0 \%)$ & $15537(43,8 \%)$ & $4388(12,4 \%)$ & $455(1,3 \%)$ \\
\hline 2001 & $35561(100,0 \%)$ & $8692(24,4 \%)$ & $1354(3,8 \%)$ & $1323(3,7 \%)$ & $258(0,7 \%)$ & $189(0,5 \%)$ & $169(0,5 \%)$ & $1447(4,1 \%)$ & $1631(4,6 \%)$ & $15677(44,1 \%)$ & $4190(11,8 \%)$ & $631(1,8 \%)$ \\
\hline 2002 & $34330(100,0 \%)$ & $8858(25,8 \%)$ & $1323(3,9 \%)$ & $1307(3,8 \%)$ & $302(0,9 \%)$ & $188(0,5 \%)$ & $171(0,5 \%)$ & $1180(3,4 \%)$ & $1626(4,7 \%)$ & $14842(43,2 \%)$ & $3927(11,4 \%)$ & $606(1,8 \%)$ \\
\hline 2003 & $32942(100,0 \%)$ & $8801(26,7 \%)$ & $1431(4,3 \%)$ & $1065(3,2 \%)$ & $416(1,3 \%)$ & $163(0,5 \%)$ & $151(0,5 \%)$ & $1097(3,3 \%)$ & $1548(4,7 \%)$ & $13854(42,1 \%)$ & $3659(11,1 \%)$ & $757(2,3 \%)$ \\
\hline 2004 & $31013(100,0 \%)$ & $8831(28,5 \%)$ & $1458(4,7 \%)$ & $958(3,1 \%)$ & $510(1,6 \%)$ & $184(0,6 \%)$ & $121(0,4 \%)$ & $992(3,2 \%)$ & $1527(4,9 \%)$ & $11168(36,0 \%)$ & $4513(14,6 \%)$ & $751(2,4 \%)$ \\
\hline 2005 & $29024(100,0 \%)$ & $8964(30,9 \%)$ & $1545(5,3 \%)$ & $978(3,4 \%)$ & $475(1,6 \%)$ & $144(0,5 \%)$ & $145(0,5 \%)$ & $1059(3,6 \%)$ & $1615(5,6 \%)$ & $8677(29,9 \%)$ & $4686(16,1 \%)$ & $736(2,5 \%)$ \\
\hline 2006 & $28480(100,0 \%)$ & $8571(30,1 \%)$ & $1439(5,1 \%)$ & $944(3,3 \%)$ & $537(1,9 \%)$ & $173(0,6 \%)$ & $172(0,6 \%)$ & $1294(4,5 \%)$ & $1692(5,9 \%)$ & $7393(26,0 \%)$ & $5327(18,7 \%)$ & $938(3,3 \%)$ \\
\hline 2007 & $27814(100,0 \%)$ & $9444(34,0 \%)$ & $1917(6,9 \%)$ & $1029(3,7 \%)$ & $579(2,1 \%)$ & $160(0,6 \%)$ & $191(0,7 \%)$ & $1322(4,8 \%)$ & $1821(6,5 \%)$ & $6208(22,3 \%)$ & $4064(14,6 \%)$ & $1079(3,9 \%)$ \\
\hline 2008 & $27430(100,0 \%)$ & $9412(34,3 \%)$ & $2188(8,0 \%)$ & $843(3,1 \%)$ & $674(2,5 \%)$ & $138(0,5 \%)$ & $179(0,7 \%)$ & $1211(4,4 \%)$ & $1884(6,9 \%)$ & $5866(21,4 \%)$ & $3851(14,0 \%)$ & $1184(4,3 \%)$ \\
\hline 2009 & $27661(100,0 \%)$ & $8982(32,5 \%)$ & $2224(8,0 \%)$ & $922(3,3 \%)$ & $764(2,8 \%)$ & $182(0,7 \%)$ & $169(0,6 \%)$ & $1552(5,6 \%)$ & $2040(7,4 \%)$ & $5895(21,3 \%)$ & $3734(13,5 \%)$ & $1197(4,3 \%)$ \\
\hline 2010 & $27099(100,0 \%)$ & $9253(34,1 \%)$ & $2520(9,3 \%)$ & $952(3,5 \%)$ & $686(2,5 \%)$ & $167(0,6 \%)$ & $212(0,8 \%)$ & $1634(6,0 \%)$ & $1977(7,3 \%)$ & $5600(20,7 \%)$ & $3125(11,5 \%)$ & $973(3,6 \%)$ \\
\hline 2011 & $26220(100,0 \%)$ & $9926(37,9 \%)$ & $2857(10,9 \%)$ & $793(3,0 \%)$ & $634(2,4 \%)$ & $144(0,5 \%)$ & $213(0,8 \%)$ & $523(2,0 \%)$ & $2034(7,8 \%)$ & $5621(21,4 \%)$ & $2499(9,5 \%)$ & $976(3,7 \%)$ \\
\hline 2012 & $27358(100,0 \%)$ & $9734(35,6 \%)$ & $3291(12,0 \%)$ & $902(3,3 \%)$ & $604(2,2 \%)$ & $150(0,5 \%)$ & $195(0,7 \%)$ & $506(1,8 \%)$ & $2110(7,7 \%)$ & $6387(23,3 \%)$ & $2246(8,2 \%)$ & $1233(4,5 \%)$ \\
\hline 2013 & $26244(100,0 \%)$ & $9196(35,0 \%)$ & $3524(13,4 \%)$ & $737(2,8 \%)$ & $553(2,1 \%)$ & $157(0,6 \%)$ & $208(0,8 \%)$ & $501(1,9 \%)$ & $2184(8,3 \%)$ & $5830(22,2 \%)$ & $2134(8,1 \%)$ & $1220(4,6 \%)$ \\
\hline 2014 & $27343(100,0 \%)$ & $9613(35,2 \%)$ & $3444(12,6 \%)$ & $805(2,9 \%)$ & $627(2,3 \%)$ & $157(0,6 \%)$ & $219(0,8 \%)$ & $533(1,9 \%)$ & $2239(8,2 \%)$ & $5978(21,9 \%)$ & $2338(8,6 \%)$ & $1390(5,1 \%)$ \\
\hline 2015 & $25641(100,0 \%)$ & $8420(32,8 \%)$ & $3770(14,7 \%)$ & $780(3,0 \%)$ & $772(3,0 \%)$ & $163(0,6 \%)$ & $184(0,7 \%)$ & $520(2,0 \%)$ & $2308(9,0 \%)$ & $5174(20,2 \%)$ & $2215(8,6 \%)$ & $1335(5,2 \%)$ \\
\hline 2016 & $25330(100,0 \%)$ & $8011(31,6 \%)$ & $4112(16,2 \%)$ & $806(3,2 \%)$ & $793(3,1 \%)$ & $142(0,6 \%)$ & $187(0,7 \%)$ & $529(2,1 \%)$ & $2288(9,0 \%)$ & $4700(18,6 \%)$ & $2243(8,9 \%)$ & $1519(6,0 \%)$ \\
\hline 2017 & $25445(100,0 \%)$ & $7833(30,8 \%)$ & $4059(16,0 \%)$ & $715(2,8 \%)$ & $928(3,6 \%)$ & $145(0,6 \%)$ & $185(0,7 \%)$ & $551(2,2 \%)$ & $2461(9,7 \%)$ & $4462(17,5 \%)$ & $2427(9,5 \%)$ & $1679(6,6 \%)$ \\
\hline 2018 & $24109(100,0 \%)$ & $6855(28,4 \%)$ & $3367(14,0 \%)$ & $714(3,0 \%)$ & $880(3,7 \%)$ & $134(0,6 \%)$ & $216(0,9 \%)$ & $740(3,1 \%)$ & $2207(9,2 \%)$ & $3607(15,0 \%)$ & $4372(18,1 \%)$ & $1017(4,2 \%)$ \\
\hline Total & $544545(100,0 \%)$ & $\begin{array}{c}167145 \\
(30,7 \%) \\
\end{array}$ & $46467(8,5 \%)$ & $\begin{array}{l}17907 \\
(3,3 \%) \\
\end{array}$ & $\begin{array}{l}11335 \\
(2,1 \%)\end{array}$ & $\begin{array}{c}3058 \\
(0,6 \%)\end{array}$ & $\begin{array}{c}3464 \\
(0,6 \%)\end{array}$ & $\begin{array}{l}20479 \\
(3,8 \%)\end{array}$ & $\begin{array}{l}36600 \\
(6,7 \%)\end{array}$ & $\begin{array}{l}152476 \\
(28,0 \%) \\
\end{array}$ & $\begin{array}{c}65938 \\
(12,1 \%)\end{array}$ & $\begin{array}{l}19676 \\
(3,6 \%) \\
\end{array}$ \\
\hline
\end{tabular}

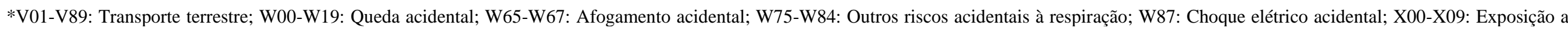
fogo acidental; X60-X84: Suicídio; X85-Y09: Homicídio; Y10-34: Acidente não especificado; Y10-Y34: Lesões de intenção indeterminada.

p-valor $<0,0001$.

Fonte: Sistema de Informação sobre Mortalidade (SIM), TABNET, São Paulo. 
No que se refere aos locais dos óbitos por causas externas 48,9\% ocorreram em hospitais, 24,8\% em outros locais, 11,9\% em domicílios, 4,5\% em outros estabelecimentos de saúde e 9,9\% foram em locais ignorados/não informados. Chama a atenção para o aumento dos óbitos em domicílio, que passou de 8,3\% em 2000 para 15,0\% em 2018 (p<0,0001).

\section{Discussão}

Neste estudo observou-se que as taxas de óbitos por causas externas no estado de São Paulo vêm apresentando tendência de queda. Embora com dados referentes a anos anteriores aos deste estudo, esta tendência também foi observada em outros países da América Latina, como no Chile, de 1997 a 2014, coeficiente angular de: -1,18/100.000 hab., IC95\%: -1,580,78 (San Martín Campos, Ponce Castro, Proboste Naranjo, Yohannessen-Vásquez, 2019) e na Colômbia, onde o risco de morrer por causas externas, nos anos estudados, foi de 81,7/100.000 habitantes, com diminuição de 47,1\% (105,94/100.000 hab. em 1998; 56,03/100.000 hab. em 2015) (Segura-Cardona \& Cardona-Arango, 2018). No estado de São Paulo esta tendência de queda já havia sido reportada, entre 2000 e 2016, passando de $14 \%$ do total dos óbitos para 7,6\% respectivamente. Em números absolutos de óbitos, as causas externas reduziram-se 33\% no período e a taxa de mortalidade (óbitos/100 mil hab.) por este tipo de causa reduziu-se 42\% (Mendes, 2019).

Embora com tendência de queda nas taxas, os homens continuam sendo os de maior risco para a mortalidade por causas externas, dado este observado em estudos internacionais, Colômbia - 86,5\% (Segura-Cardona \& Cardona-Arango, 2018), Chile - 85,3\% (San Martín Campos, Ponce Castro, Proboste Naranjo, Yohannessen-Vásquez, 2019), SucumbíosEquador -80,7\% (Andrial, 2017), Etiópia - 67,1\% (Gelave, Tessema, Tariku , Abera, Gebru , Assefa.......Lakew, 2018), e nacionais, Tocantins -82,46\% (Messias, Bandeira, Lopes, Silva, Curado, 2018), região Sul (estados de Santa Catarina, Paraná e Rio Grande do Sul) - 56,94\% (Preis, Lessa, Tourinho, dos Santos, 2018), Joinville-83,6\% (Cardoso, Gaertner, Haritsch, Henning, Kropiwiec, Franco, 2020).

Neste estudo a maior proporção de óbitos foi entre os indivíduos de 15 a 29 anos, porém neste grupo, ao longo dos anos, observou-se tendência de queda expressiva, por outro lado verificou-se ascensão no grupo dos idosos. Vários estudos apontam serem os jovens os mais expostos à mortalidade por causas externas: Chile de 25 a 44 - 44,8\% (San Martín Campos, Ponce Castro, Proboste Naranjo, Yohannessen-Vásquez, 2019), Tocantins de 20 a 29 anos - 24,85\% (Messias, Bandeira, Lopes, Silva, Curado, 2018)), Região Sul de 20 a 39 anos - 42,73\% (Preis, Lessa, Tourinho, dos Santos, 2018), Joinville de 25-39 anos (30,5\%) (Cardoso, Gaertner, Haritsch, Henning, Kropiwiec, Franco, 2020). Ressalta-se que estudo nacional, realizado de 1996 a 2013 revelou tendência de aumento da mortalidade para os idosos passando de 94,2/100 mil habitantes para 103,6/100 mil habitantes, correspondendo a um aumento de 10,0\% (Carmo, Souza Nery Vilela, Martins Filho, 2017).

A maioria dos óbitos ocorreu em indivíduos de raça/cor branca e isto guarda relação com o perfil populacional das regiões do país. Na região Sul 86,21\% dos óbitos foram em indivíduos brancos (Preis, Lessa, Tourinho, dos Santos, 2018), já em Rondônia e Bahia foram em pardos, respectivamente, 53,9\% e 71,5\% (Pereira, de Araújo, Moreira, Figueiredo, 2020; da Silva, Vieira, Nery, Abreu, Silva, de Jesus, 2018). Ressalta-se que, em 2010, no estado de São Paulo, 63,9\% se autoautodeclaram brancos (Fundação Sistema Estadual de Análises de Dados-SEADE, 2021).

Neste estudo, embora a mortalidade por causas externas tenha sido maior em indivíduos de 4 a 7 anos de escolaridade, o aumento na proporção de óbitos (2000-2018) ocorreu entre indivíduos com 8 anos ou mais de escolaridade. Pesquisa que apresentou as desigualdades de gênero na mortalidade por causas externas no Brasil, corrobora com os achados deste estudo, em homens - 28,6\% dos óbitos ocorreram em indivíduos com escolaridade menor ou igual oito anos de escolaridade e 39,4\% naqueles com mais de oito anos, mulheres $-6,7 \%$ dos óbitos ocorreram em mulheres com escolaridade menor ou igual oito anos de escolaridade e 12,4\% naquelas com mais de oito anos; os autores concluíram que homens com maior escolaridade têm risco $20 \%$ maior e mulheres, $44 \%$ maior, que as respectivas categorias com menor nível educacional (de Moura, Gomes, 
Falcão, Schwarz, das Neves, Santos, 2015). Embora com tendência à diminuição, ainda há um grande número de ignorados (35,7\% do total) e tal fato pode interferir na análise precisa dos dados, ressalta-se que apesar das instruções claras e padronizadas para o preenchimento dos instrumentos de coleta de dados dos sistemas de informação brasileiros, a completitude ainda é um desafio (Ribeiro \& Njaine, 2020).

Em relação às causas (CID 10) dos óbitos notou-se que as mortes por queda acidental aumentaram proporcionalmente no período em relação aos outros tipos, isto pode estar relacionado ao envelhecimento populacional, uma vez que este segmento da população é o mais exposto à tipo de agravo (Wingerter, Barbosa, Moura, Maciel, Alves, 2020). Chama a atenção também o aumento dos óbitos por acidentes de transporte, riscos acidentais à respiração e suicidio. Estudos relacionados à temática divergem na ordem de magnitude em relação ao tipo de causas. Na Etiópia as principais causas foram afogamento acidental e submersão $(21,8 \%)$, queda acidental $(18,1 \%)$ e acidentes relacionados ao transporte $(18,0 \%)$ (Gelave, Tessema, Tariku , Abera, Gebru , Assefa, Lakew, 2018), no Chile, acidentes de trânsito (29,3\%) e lesões auto-inflingidas (25,7\%) (San Martín Campos, Ponce Castro, Proboste Naranjo,Yohannessen-Vásquez, 2019), em Tocantins, acidentes de transporte (43,92\%) e agressões (29,98\%) (Messias, Bandeira, Lopes, Silva, Curado, 2018), em Rondônia observou-se um aumento anual nos óbitos por acidentes de trânsito de 2,1\% (Pereira, de Araújo, Moreira, Figueiredo, 2020) e em Joinville, acidentes de transportes (40,0\%) e agressões (30,5\%) (Cardoso, Gaertner, Haritsch, Henning, Kropiwiec, Franco, 2020). Observa-se que os acidentes de transportes configuram como uma das principais causas de óbitos entre todos os estudos relatados. Neste estudo observou-se tendência de queda em relação dos óbitos por agressões (homicídios), dado este também observado na Colômbia de 1998-2015 (redução de 59,3\%) (Segura-Cardona \& Cardona-Arango, 2018) e Rondônia (decréscimo anual de 1,98\%) (Pereira, de Araújo, Moreira, Figueiredo, 2020).

O hospital foi o principal local de óbitos por causas externas, dado este, também evidenciado em estudo realizado na região sul do país, (40,70\%) (Preis, Lessa, Tourinho, dos Santos, 2018).

Não obstante às contribuições feitas nesta pesquisa, algumas limitações deste estudo devem ser consideradas, dados obtidos de fontes secundárias podem ter problemas relacionados a qualidade, tais como, falta de preenchimento de alguns campos da declaração de óbito e codificação, porém em se tratando de mortalidade é uma das fontes de informação mais seguras, universal e de boa confiabilidade.

A despeito das limitações supracitadas deve-se ressaltar que estudos de séries temporais sobre mortalidade podem ser importantes ferramentas epidemiológicas para a formulação de hipóteses explicativas, identificação de grupos de maior vulnerabilidade ao agravo e avaliação indireta da efetividade das políticas públicas.

\section{Conclusão}

Os resultados do presente estudo documentam uma tendência de queda nas taxas de mortalidade por causas externas no estado de São Paulo, período (2000-2018).

Embora o sexo masculino ainda apresente a maior taxa de óbitos, este extrato, foi o que apresentou tendência de queda mais expressiva.

Em relação as faixas etárias houve tendência de queda em todas as idades, exceção ao grupo de idosos, sendo esta mais expressiva entre as pessoas de 15 a 29 anos.

Òbitos em individuos de raça/cor branca foram predominantes em todo o periodo estudo, sendo os solteiros o grupo com maior diminuição de óbitos na série histórica. Já em relação a escolaridade observou-se diminuição das proporções de óbitos nos indivíduos com menos escolaridade, em contrapartida, aumento em pessoas com mais anos de estudo. 
Em relação às causas observou-se aumento nas proporções óbitos por quedas, acidentes por transporte terrestre, outros riscos acidentais à respiração e suicídio e diminuição por homicídio, sendo o hospital o local de maior concentração dos óbitos.

Sugere-se que trabalhos desta natureza devam ser incentivados e desenvolvidos, inclusive em outras regiões do país, faz-se importante também acompanhar as tendências da mortalidade por causas externas em anos posteriores ao deste estudo, para verificar, inclusive, o impacto da pandemia do COVID-19 nestes indicadores.

\section{Referências}

Andrial, L. H. (2017). Epidemiología de los casos trabajados en el Centro de Investigación de Ciencias Forenses de Sucumbíos (Ecuador), año 2016. Cuad Med Forense, 23(1-2),24-34. https://scielo.isciii.es/scielo.php?script=sci_arttext\&pid=S1135-76062017000100024\&lng=es\&nrm=iso\&tlng=es

Calazans, J. A. \& Queiroz B. L. (2020). The adult mortality profile by cause of death in 10 Latin American countries (2000-2016). Rev Panam Salud Publica, 44. doi.org/10.26633/RPSP.2020.1

Cardoso S., Gaertner M. H. C. N., Haritsch L., Henning E., Kropiwiec M. V., \& Franco S. C. (2020). Perfil e evolução da mortalidade por causas externas em Joinville (SC), 2003 a 2016. Cad. Saúde Colet., 28(2),189-200. doi.10.1590/1414-462X202028020115.

Carmo E. A., Souza T. S., Nery A. A., Vilela A. B. A., \& Martins Filho I. E. (2017). Tendência da mortalidade por causas externas em idosos. J Nurs UFPE, 11(Suppl. 1), 374-82. 10.5205/1981-8963-v11i1a11918p374-382-2017

Core Team (2018). R: A language and environment for statistical computing. Vienna, Austria: R Foundation for Statistical Computing. https://www.Rproject.org/.

Da Silva R. A., Vieira C. X., Nery A. A., Abreu F. S., Silva N. A., \& de Jesus L. R. (2018). Mortality due to external causes in youth in state of Bahia. Rev Fun Care Online, 10(1), 46-51. 10.9789/2175-53612018.v10i1.46-51.

De Moura E. C., Gomes R., Falcão M. T. C., Schwarz E., das Neves A. C. M., \& Santos W. (2015). Gender inequalities in external cause mortality in Brazil, 2010. Ciência \& Saúde Coletiva, 20(3),779-788. 10.1590/1413-81232015203.11172014.

Fundação Sistema Estadual de Análises de Dados -SEADE (2010). Retratos de São Paulo: População. http://produtos.seade.gov.br/produtos/retratosdesp/view/index.php?indId=5\&temaId=1\&locId=1000

Gelaye K., Tessema F., Tariku B., Abera S. F., Gebru A. A., Assefa N. \& Lakew Y. (2018). Injury-related gaining momentum as external causes of deaths in Ethiopian health and demographic surveillance sites: evidence from verbal autopsy study. Global Health Action,11, 1430669. $10.1080 / 16549716.2018 .1430669$

Lima- Costa, M. F. \& Barreto, S. M. (2003). Tipos de estudos epidemiológicos: Conceitos básicos e aplicações na área do envelhecimento. Epidemiologia e Serviços de Saúde, 12 (4). 49742003000400003

Malta D. C., Minayo M. C. S., Soares Filho A. M., da Silva M. M. A., Montenegro M. M. S., Ladeira R. M. \& Naghavi M. (2017). Mortalidade e anos de vida perdidos por violências interpessoais e autoprovocadas no Brasil e Estados: análise das estimativas do Estudo Carga Global de Doença, 1990 e 2015. Rev. bras. epidemiol.,20(Suppl 1),142-156. 10.1590/1980-5497201700050012.

Marques S. H. B., de Souza A. C., Vaz A., Pelegrini A. H. W., \& Linch G. F. C. (2017). Mortalidade por causas externas no Brasil de 2004 a 2013.Revista Baiana de Saúde Pública, 41(2), 394-409. 10.22278/2318-26602017v41.n2a2368.

Mendes J. D. V. (2019). Mortalidade por causas externas no Estado de São Paulo de 2000 a 2016. Bol. Epidemiol. Paul. (BEPA),16(185),11-24. https://pesquisa.bvsalud.org/portal/resource/pt/biblio-1023367

Messias M. M., Bandeira J. R., Lopes A. B., Silva L. L. D., Curado P. F. (2018). Mortalidade por causas externas: revisão dos dados do Sistema de Informação de Mortalidade. Rev Soc Bras Clin Med.,16(4),218-21. http://www.sbcm.org.br/ojs3/index.php/rsbcm/article/view/374/336

Minayo M. C. S., de Souza E. R., da Silva M. M. A., \& de Assis S. G. (2018). Institucionalização do tema da violência no SUS: avanços e desafios. Ciência \& Saúde Coletiva, 23(6),2007-2016. 10.1590/1413-81232018236.04962018.

Ministério da Saúde (2003). Política nacional de redução da morbimortalidade por acidentes e violências: Portaria MS/GM n. ${ }^{\circ} 737$ de 16 de maio de 2001. Brasília: Ministério da Saúde. https://bvsms.saude.gov.br/bvs/publicacoes/politica_nacional_reducao_morbimortalidade.pdf

Ministério da Saúde (2019). Departamento de Informática do Sistema Único de Saúde (DATASUS). Sistema de informação sobre mortalidade. http://tabnet.datasus.gov.br/cgi/deftohtm.exe?sim/cnv/ext10br.def.

Organização Mundial da Saúde. (2000). CID 10: classificação estatística internacional de doenças e problemas relacionados à saúde. Décima Revisão. (8a ed.). São Paulo: Edusp.

Pereira P. P. S., de Araújo L. X., Moreira K. F. A., \& Figueiredo A. C. M. G. (2020). Mortalidade por causas externas no estado de Rondônia: análise de série temporal de 1999 a 2015. Rev Fun Care Online, 12,270-275. 10.9789/2175-5361.rpcfo.v12.8501.

Preis L. C. C., Lessa G., Tourinho F., \& Dos Santos J. L. G. (2018). Epidemiologia da mortalidade por causas externas no período de 2004 a 2013 . Revista de Enfermagem UFPE, 12(13),716-728. 10.5205/1981-8963-v12i3a230886p716-728-2018. 
Research, Society and Development, v. 10, n. 9, e15101018627, 2021

(CC BY 4.0) | ISSN 2525-3409 | DOI: http://dx.doi.org/10.33448/rsd-v10i10.18627

Reichenheim M. E., de Souza E. R., Moraes C., de Mello Jorge M. H. P., da Silva C. M. F. P., \& Minayo M. C. S. (2011). Violência e lesões no Brasil: efeitos, avanços alcançados e desafios futuros. The Lancet, 4,377(9781),1962-75. 10.1016/S0140-6736(11)60053-6.

Rede Interagencial de Informação para a Saúde (2008). Indicadores básicos para a saúde no Brasil: conceitos e aplicações. Brasília: Organização PanAmericana da Saúde. http://www.ripsa.org.br/2014/10/30/indicadores-basicos-para-a-saude-no-brasil-conceitos-e-aplicacoes-livro-2a-edicao-2008-2

Ribeiro A. P. \& Njaine K. (2020). A gestão em saúde na prevenção e atenção às situações de violência. In: Njaine K, de Assis SG, Constantino P, Avanci, JQ. (orgs). Impactos da Violência na Saúde. Rio de Janeiro: FIOCRUZ (pp. 367- 385). 10.7476/9786557080948.

San Martín Campos C., Ponce Castro J., Proboste Naranjo A., \& Yohannessen-Vásquez K. (2019). Evolución temporal de la mortalidad prematura por causas externas en Chile entre 1997-2014. Rev Esp Salud Pública, 93:20, e201905028. https://scielo.isciii.es/scielo.php?script=sci_arttext\&pid=S113557272019000100064

Secretaria de Estado de Saúde São Paulo (2021). Informações de Saúde -TABNET https://www.saude.sp.gov.br/links/informacoes-de-saude-tabnet.

Segura-Cardona A. \& Cardona-Arango D. (2018). Mortalidad y años potenciales de vida perdidos por causas externas: Colombia 1998-2015. Univ. Salud., 20(2),149-159. dx.doi.org/10.22267/rus.182002.119

Wingerter D. G., Barbosa I. R., Moura L. K. B., Maciel R. F., \& Alves M. S. C. F. (2020). Mortalidade por queda em idosos: uma revisão integrativa. Revista Ciência Plural, 6(1), 119-136. 10.21680/2446-7286.2020v6n1ID18366.

World Health Organization. (2014). Injuries and violence: the facts 2014. Geneva: WHO. https://apps.who.int/iris/bitstream/handl e/10665/149798/9789241508018_eng.pdf?sequence=1\&isAllowed=y 